\title{
Efficient Irradiance Normal Mapping
}

\author{
Ralf Habel and Michael Wimmer \\ Institute of Computer Graphics and Algorithms \\ Vienna University of Technology, Austria
}
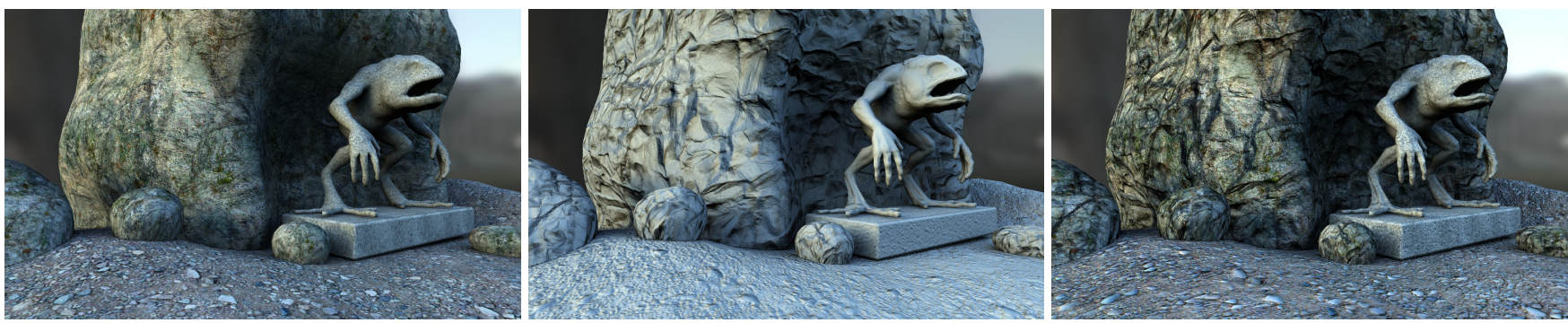

Figure 1: A typical game scene using standard light mapping (left) and irradiance normal mapping using the $\mathcal{H}$-basis without (middle) and with (right) textured albedo.

\begin{abstract}
Irradiance normal mapping is a method to combine two popular techniques, light mapping and normal mapping, and is used in games such as Half-Life 2 or Halo 3. This combination allows using low-resolution light caching on surfaces with only a few coefficients which are evaluated by normal maps to render spatial high-frequency changes in the lighting. Though there are dedicated bases for this purpose such as the Half-Life 2 basis, higher order basis functions such as quadratic Spherical Harmonics are needed for an accurate representation. However, a full spherical basis is not needed since the irradiance is stored on the surface of a scene.
\end{abstract}

In order to represent the irradiance signals efficiently, we propose a novel polynomial, hemispherically orthonormal basis function set that is specifically designed to carry a directional irradiance signal on the hemisphere and which makes optimal use of the number of coefficients. To compare our results with previous work, we analyze the relations and attributes of previously proposed basis systems and show that 6 coefficients are sufficient to accurately represent an irradiance signal on the hemisphere. To create the necessary irradiance signals, we use Spherical Harmonics as an intermediate basis due to their fast filtering capabilities.

CR Categories: I.3.7 [COMPUTER GRAPHICS]: ThreeDimensional Graphics and Realism-; I.3.3 [COMPUTER GRAPHICS]: Color, Shading, Shadowing and Texture-;

Keywords: real-time rendering, irradiance, caching, lightmap, normal mapping

\section{Introduction}

Light mapping and normal mapping are the most successful shading techniques used in commercial games and applications because they require only few resources and result in a significant increase in the quality of the rendered image. While light mapping stores global, low-frequency illumination at sparsely sampled points in the scene, normal maps provide local, high-frequency shading variation at a high resolution. The problem with combining the two methods is that light maps store lighting information for only one normal direction (the base surface normal) and therefore cannot be evaluated using the normals stored in a normal map. To overcome this problem, the irradiance (i.e., the incoming radiance integrated over the hemisphere) for all possible normal map directions has to be calculated and stored for each illumination sample point on the surface.

Because the irradiance signal is low frequency in its directionality, it can be well represented by lower order basis functions. As shown by Ramamoorthi and Hanrahan [Ramamoorthi and Hanrahan 2001] and Basri and Jacobs [Basri and Jacobs 2000], using Spherical Harmonics up to the quadratic band ( 9 coefficients per color) is sufficient to represent an irradiance signal over the whole sphere of normal directions (a so-called irradiance environment map) with only a small average error of less than 3 percent for a typical irradiance signal. This representation was adopted in games (e.g., [Chen and Liu 2008]) to store spatially varying irradiance at surface samples using Spherical Harmonics light maps. However, while Spherical Harmonics work well, irradiance normal mapping on surfaces actually only requires a hemispherical signal - the information contained in the inner hemisphere is never used.

In this paper, we therefore propose a new hemispherical basis called $\mathcal{H}$-basis, which is designed to represent irradiance signals over the hemisphere of possible surface normals. The main advantage of the $\mathcal{H}$-basis is that it requires the same amount of coefficients than Spherical Harmonics to represent irradiance signals on the hemisphere and less error than other hemispherical bases, while keeping the main advantages of Spherical Harmonics on a sphere: (1) It is polynomial and therefore fast to evaluate. (2) Since it is closely related to Spherical Harmonics, a signal given in Spherical Harmonics can be converted to the $\mathcal{H}$-basis using a sparse matrix. (3) It is orthonormal and therefore any general function can be easily projected into the basis without requiring a least-squares fit if it is used for other purposes. (4) It is guaranteed that a linear interpolation of the coefficients results in a linear interpolation of the evaluation of the basis, which is especially important if the irradiance is stored sparsely such as on a per vertex basis or very small coefficient maps. 


\section{Previous Work}

Several basis systems have been proposed to represent hemispherical functions in general as well as specifically for irradiance normal mapping. We are not considering spherical wavelets [Schröder and Sweldens 1995] since only a very small number of coefficients are needed and an iterative fitting of millions of functions is prohibitively expensive. Also, we will not discuss Spherical Harmonics separately as they are covered extensively in the literature([Sloan et al. 2002],[Ramamoorthi and Hanrahan 2001],[Green 2003],[Sloan 2008]). A similar problem of calculating the irradiance occurs when combining Spherical Harmonics PRT with normal mapping [Sloan 2006], though the radiance is not precomputed but calculated with the precomputed transfer functions. Since only a minimal number of coefficients are considered, numerical stability and compression artifacts [Lam et al. 2006] are comparable to standard light mapping.

Ultimately, the ratio of accuracy to number of used coefficients defines the effectiveness of a basis function set, independent of the irradiance functions represented.

\subsection{Half-Life 2 Basis and Directional Occlusion}

The Half-Life 2 Basis [McTaggert 2004] [Green 2007], is a function set that is orthonormal over the upper unit hemisphere $\Omega_{+}$. It is defined by three cosine lobes spanned by the vectors $\overrightarrow{h_{i}}$

$$
\left(\begin{array}{c}
\frac{-1}{\sqrt{6}} \\
\frac{-1}{\sqrt{2}} \\
\frac{1}{\sqrt{3}}
\end{array}\right)\left(\begin{array}{c}
\frac{-1}{\sqrt{6}} \\
\frac{1}{\sqrt{2}} \\
\frac{1}{\sqrt{3}}
\end{array}\right)\left(\begin{array}{c}
\sqrt{\frac{2}{3}} \\
0 \\
\frac{1}{\sqrt{3}}
\end{array}\right) .
$$

The explicit evaluation $E_{\mathrm{HL} 2}$ is executed through the dot products of the evaluation direction $\vec{n}$ and the basis directions weighted by their coefficients:

$$
E_{\mathrm{HL} 2}(\vec{x})=\sum_{i=1}^{3} \sqrt{\frac{3}{2 \pi}} c_{i} \vec{n} \cdot \overrightarrow{h_{i}}
$$

with the basis coefficients $c_{i}$ and the normalization factor $\sqrt{\frac{3}{2 \pi}}$. Though defined on the hemisphere, the three basis functions are, due to the linearity of the basis, a rotated and hemispherically normed version of linear Spherical Harmonics, which also consist of three cosine lobes. Actually, due to linearity, any three mutually orthogonal vectors define an equivalent basis, although the given configuration causes the coefficients to be mostly positive, circumventing a range compression if the coefficients are saved in 8 bit colors.

Expression 2 can be simplified by extracting $\vec{x}$ as a common factor, which simplifies the evaluation to a single dot product with the (non-normalized) vector:

$$
\vec{o}=\sum_{i=1}^{3} \sqrt{\frac{3}{2 \pi}} c_{i} \overrightarrow{h_{i}}
$$

This formulation is also called Directional Occlusion. Unfortunately, if a trichromatic signal is represented, each color requires a separate Directional Occlusion vector $\vec{o}$, and thus three dot products have to be calculated while the Half-Life 2 Basis needs to be evaluated only once, giving no advantage in the trichromatic case because 3 dot products have to be performed in both cases. However, the Directional Occlusion formulation shows that the HalfLife 2 basis is fully equivalent to Directional Occlusion and both bases can transport one general cosine lobe.

\subsection{Hemispherical Harmonics and Makhotkin Basis}

Hemispherical Harmonics (HSH) [Gautron et al. 2004] and the Makhotkin basis [Makhotkin 1996] are hemispherically orthonormal bases that are constructed by mapping the negative pole of the sphere at $(0,0,-1)$ to the border of the hemisphere, contracting the spherical orthonormal functions to hemispherical ones through a shifting operation without destroying their orthogonality. This shifting is generated by replacing the $\cos \theta$ term in the definition with $2 \cos \theta-1$.

In the case of Makhotkin, Jacobi polynomials are shifted, whereas Hemispherical Harmonics consist of shifted Spherical Harmonics. Due to this contraction, the basis functions become strongly nonpolynomial and therefore expensive to evaluate as they contain several square roots and divisions. This is important since for irradiance caching, the basis functions are evaluated directly in the pixel shader. Also, due to the shifting, all basis functions are either 0 or constant at the border of the hemisphere, causing severe errors localized at the border. This effect diminishes with higher bands, but is dominant if only two or three bands are used since only one constant color can be represented at the border of the hemisphere. Due to their spherical origin, their band structure is the same as Spherical Harmonics.

\subsection{Zernike Basis}

Zernike polynomials, an orthonormal polynomial set on the unit circle, have been adapted to the hemisphere by Koenderink et al. [Koenderink et al. 1996] to represent BRDFs. However, the adaptation introduces square roots and divisions into the basis functions, which results in a high evaluation cost compared to purely polynomial bases. Due to their two-dimensional origin, they also feature a different band structure, having only two basis functions in the first band and three in the second band, resulting in 3 coefficients up to the first band and 6 coefficients up to the second band. This makes this basis interesting for an irradiance signal representation with only a few coefficients.

Compared to shifted hemispherical bases, the Zernike basis does not suffer from severe errors at the border of the hemisphere because the basis functions vary at the border of the hemisphere.

\section{Creating the Directional Irradiance}

The irradiance of a surface point $\vec{x}$ with surface normal $\vec{n}$ is defined as

$$
E(\vec{x}, \vec{n})=\int_{\Omega^{+}} L(\vec{x}, \omega)(\vec{n} \cdot \vec{\omega}) d \vec{\omega} .
$$

A representation of the signal $E(\vec{x}, \vec{n})$ is stored at sparse sample points $\vec{x}$, for example at object vertices or at the texels of a lowresolution texture map. The signal is defined for all normal vectors $\vec{n}$ in the upper hemisphere of the point $\vec{x}$. In practice, the hemisphere is defined in tangent space, i.e., around the interpolated surface normal and (bi)tangent vector.

Similar to light mapping, we first need to calculate the radiance and derive the irradiance in a precomputation step.Any method that creates a radiance estimate, such as shadow mapping, photon mapping [Jensen 1996], or path tracing [Kajiya 1986] can be used. In the figures shown in this paper, the radiance solution was calculated by ray tracing an importance-sampled HDR environment map, which allows testing all bases under high dynamic range conditions and arbitrarily complex radiance signals. 
Given the radiance $L$, creating $E(\vec{x}, \vec{n})$ (equation 4) corresponds to filtering $L$ with a diffuse kernel. Doing this in Euclidean or spherical coordinates is prohibitively expensive due to the high cost of every single convolution and the high number of surface sample points for which this has to be done (usually tens of millions in current game levels).

Instead, we use Spherical Harmonics as an intermediate basis. Ramamoorthi et al. [Ramamoorthi and Hanrahan 2001] and Basri [Basri and Jacobs 2000] show how to efficiently carry out equation 4 in this basis. Furthermore, they show that an irradiance signal represented in Spherical Harmonics requires only 3 bands ( 9 coefficients) to achieve an average error below $3 \%$.

We rotate each sampled radiance signal into tangent space and expand it into the Spherical Harmonics coefficients $s_{m}^{l}$ by integrating against the Spherical Harmonics basis functions $Y_{m}^{l}$ over the complete sphere $\Omega$ :

$$
s_{m}^{l}=\int_{\Omega} L(\vec{\omega}) Y_{m}^{l}(\vec{\omega}) d \vec{\omega}
$$

Since this has to be done for a small number of coefficients only, it is much faster than a direct convolution. At this stage, we can also apply a number of different filtering methods such as described in [Sloan 2008] to remove potential ringing artifacts. The FunkHecke Theorem [Groemer 1996] states that the diffuse convolution (equation 4) can be carried out directly in Spherical Harmonics by simply scaling the coefficients. Following [Basri and Jacobs 2000], we multiply the coefficients in each band $l$ with the corresponding factor $a^{l}$ :

$$
\begin{array}{rr}
a^{0}=1 & a^{1}=\frac{2}{3} \\
a^{2}=\frac{1}{4} & a^{3}=0 \\
a^{4}=-\frac{1}{24} &
\end{array}
$$

to arrive at the irradiance signal represented in Spherical Harmonics:

$$
E_{\mathrm{SH}}(\vec{n})=\sum_{l} \sum_{m} s_{m}^{l} Y_{m}^{l}(\vec{n})
$$

Please note that because $a^{3}=0$, the 3 rd band never has to be calculated in the expansion. Since also the factor $a^{4}$ as well as all higher band factors is very small, the first three Spherical Harmonics bands are sufficient to represent the irradiance signal with a high accuracy for most practical purposes, and this is the reason why a Spherical Harmonics representation is so efficient.

If storing 9 coefficients (i.e., 27 in the trichromatic case) at each surface sample point is acceptable, we can calculate the irradiance at any surface point by directly evaluating the Spherical Harmonics basis functions using a normal looked up from the normal map and using the interpolated coefficients to evaluate equation 7. However, we are not making the most efficient use of the coefficients since the functions are only evaluated on the hemisphere $\Omega_{+}$, and not the full sphere. The created directional irradiance signals can be better represented in a hemispherical basis. In the following section, we propose a hemispherical basis that is polynomial and orthogonal, and requires less coefficients to represent irradiance signals with a comparable error to Spherical Harmonics.

\section{$4 \mathcal{H}$-Basis}

Since a Spherical Harmonics representation of irradiance signals is so successful, we want to stay as close to the Spherical Harmonics basis functions as possible when designing our new basis, yet use the coefficients more efficiently. Also, avoiding any nonpolynomial basis functions circumvents high evaluation costs, as the basis functions have to be evaluated for each fragment.

The upper hemisphere $\Omega_{+}$that carries the signal $E(\vec{x}, \vec{n})$ is a closed half-space of the full sphere, separated by the plane at $z=0$. In other words, with $\vec{n}$ given in spherical coordinates $(\theta, \phi)$, the range of the latitudinal parameter reduces from $\theta=0 . . \pi$ to $\theta=0 . . \pi / 2$, while the longitudinal parameter range stays unmodified at $\phi=$ $0 . .2 \pi$.

Our goal is to choose a set of hemispherically orthogonal elements of the polynomial Hilbert space [Sansone 1991] that can best represent a general hemispherical irradiance signal. First, we observe that since the longitudinal parameter range does not change, all Spherical Harmonics basis functions that are symmetric to the $z=0$ plane are automatically orthogonal over the upper hemisphere $\Omega_{+}$, and we can thus use them without modification. Considering the first three bands, these functions are $Y_{0}^{0}, Y_{-1}^{1}, Y_{1}^{1}, Y_{-2}^{2}$ and $Y_{2}^{2}$ (see Figure 2). Note that in the Euclidean representation of Spherical Harmonics, these are all functions that do not explicitly depend on the value of $z$.

For the latitudinal part, where the parameter range is halved, we shift $Y_{0}^{1}$ to the hemisphere analogous to the HSH and Makhotkinbasis, replacing $\cos \theta$ with $2 \cos \theta-1$. Since $\mathrm{HSH}$ is created through shifting the Spherical Harmonics to the hemisphere, this is the same function as the HSH basis function with $l=1, m=0$. Also, the Zernike basis contains the negative of this function in its second band with $l=0, n=2$. Remarkably, without any explicit orthogonalization, all functions are still orthogonal over the upper hemisphere $\Omega_{+}$, which was the initial goal. Normalization of all functions to the hemisphere results in the following set of basis functions for the $\mathcal{H}$-basis:

$$
\begin{aligned}
H^{1} & =\frac{1}{\sqrt{2 \pi}} \\
H^{2} & =-\sqrt{\frac{3}{2 \pi}} \sin \phi \sin \theta=-\sqrt{\frac{3}{2 \pi}} y \\
H^{3} & =\sqrt{\frac{3}{2 \pi}}(2 \cos \theta-1)=\sqrt{\frac{3}{2 \pi}}(2 z-1) \\
H^{4} & =-\sqrt{\frac{3}{2 \pi}} \cos \phi \sin \theta=-\sqrt{\frac{3}{2 \pi}} x \\
H^{5} & =\frac{1}{2} \sqrt{\frac{15}{2 \pi}} \sin 2 \phi \sin ^{2} \theta=\sqrt{\frac{15}{2 \pi}} x y \\
H^{6} & =\frac{1}{2} \sqrt{\frac{15}{2 \pi}} \cos 2 \phi \sin ^{2} \theta=\frac{1}{2} \sqrt{\frac{15}{2 \pi}}\left(x^{2}-y^{2}\right)
\end{aligned}
$$

Analogous to Spherical Harmonics, we can choose up to what polynomial degree (with respect to the Euclidean representation) we want to approximate the irradiance signal. Using only the first 4 coefficients corresponds to the constant and linear basis functions. We call this reduced basis $\mathcal{H}_{4}$, and refer to the full basis, including the two quadratic functions, with $\mathcal{H}_{6}$ (not to be confused with the basis functions $H^{i}$ ). Note that this allows the option to choose the number of evaluated functions at run-time, simply not evaluating the two quadratic basis functions.

The complete $\mathcal{H}$-basis is visualized in Figure 2. While most functions look very similar to their SH counterparts, just restricted to the upper hemisphere, it is interesting to take a closer look at $H^{3}$, which results from shifting $Y_{0}^{1}$. Intuitively speaking, the shifting operation increases the frequency content of this function, it has one more "mode" than the original $Y_{0}^{1}$ restricted to the hemisphere would have. It rather resembles $Y_{0}^{2}$ restricted to the hemisphere. So 


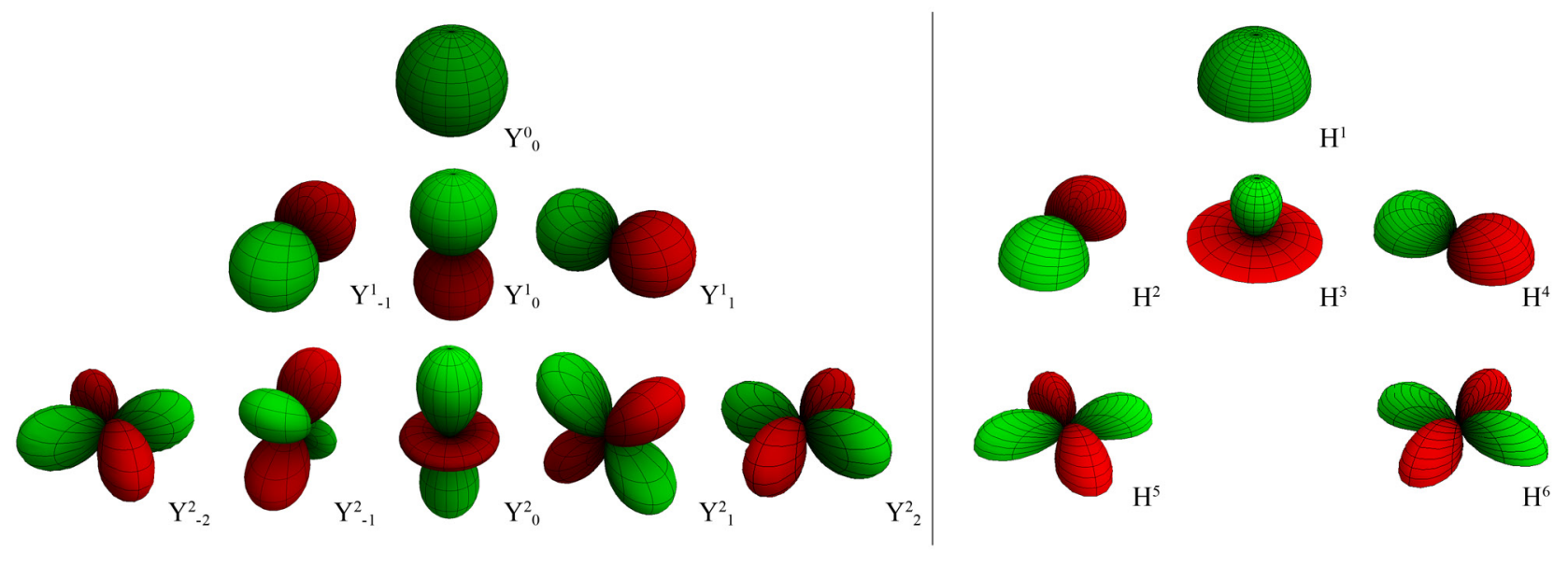

Figure 2: Spherical Harmonics basis functions (left) compared to the $\mathcal{H}$-basis functions (right).

basically, through the restriction to the hemisphere, fewer functions can provide a more accurate fit to a given signal, which explains the good accuracy shown later at a lower coefficient count. Compared to a hemispherical projection of Spherical Harmonics as proposed in [Sloan et al. 2003], $Y_{0}^{1}$ is also the basis function that can be best fitted with other Spherical Harmonics basis functions and can be omitted. Yet, a high number of basis functions that are not orthogonal to $Y_{0}^{1}$ is needed so this approach is only possible for signals represented using more than 3 bands.

The linear basis functions can also be constructed by building the proper duals [Sloan et al. 2003] of the Spherical Harmonics basis functions on the hemisphere and therefore, hemispherically leastsquare projected linear Spherical Harmonics have the same error as the $\mathcal{H}_{4}$-basis if only the upper hemisphere is used. Yet, the $\mathcal{H}_{4}$ basis is orthogonal which makes the projection into the $\mathcal{H}$-basis simpler.

\subsection{Expansion into the $\mathcal{H}$-Basis}

In Section 3, we have shown how to calculate the SH representation of an irradiance signal. To transform such a signal into the $\mathcal{H}$-basis, we calculate the transformation matrix by projecting the Spherical Harmonics up to $l=2$ into the $\mathcal{H}$-basis, resulting in the transformation matrix $T_{h}$

$$
\left(\begin{array}{ccccccccc}
\frac{1}{\sqrt{2}} & 0 & \frac{1}{2} \sqrt{\frac{3}{2}} & 0 & 0 & 0 & 0 & 0 & 0 \\
0 & \frac{1}{\sqrt{2}} & 0 & 0 & 0 & \frac{3}{8} \sqrt{\frac{5}{2}} & 0 & 0 & 0 \\
0 & 0 & \frac{1}{2 \sqrt{2}} & 0 & 0 & 0 & \frac{1}{4} \sqrt{\frac{15}{2}} & 0 & 0 \\
0 & 0 & 0 & \frac{1}{\sqrt{2}} & 0 & 0 & 0 & \frac{3}{8} \sqrt{\frac{5}{2}} & 0 \\
0 & 0 & 0 & 0 & \frac{1}{\sqrt{2}} & 0 & 0 & 0 & 0 \\
0 & 0 & 0 & 0 & 0 & 0 & 0 & 0 & \frac{1}{\sqrt{2}}
\end{array}\right)
$$

By multiplying the Spherical Harmonics coefficient vector with $T_{h}$, the $\mathcal{H}$-basis coefficient vector containing all $h_{i}$ is created. Because we stayed very close to the basis functions of Spherical Harmonics, the matrix is sparse and poses no considerable calculation overhead. If the irradiance is available with SH coefficients up to $l=4$ (with all $l=3$ coefficients 0 ), the $l=4$ coefficients can also be projected into the $\mathcal{H}$-basis with a matrix given in Appendix A. In practice, this only performs imperceptible corrections to the signal and is not necessary in almost all cases.

\subsection{Run-Time Evaluation}

Up to now, we have created the directional irradiance on the surfaces in tangent space through a precomputation step. The necessary coefficients are transported on a per vertex basis or using coefficient texture maps. In the trichromatic case, this results in 4 or 6 additional colors per vertex or texture maps. If tangent space normal maps are used, the irradiance is calculated by explicitly evaluating the basis functions in the direction of the looked up normal $\vec{n}$, weighted by the interpolated coefficients

$$
E_{H}(\vec{n})=\sum_{i=1}^{n} h_{i} H^{i}(\vec{n})
$$

for every surface point. For the final color or emitted radiance, $E_{H}$ can be multiplied by a material coefficient or a lookup from an albedo texture.

\subsection{Directional Irradiance Tangent Space}

In almost all practical cases that use texture maps instead of pervertex caching, a second set of texture coordinates that creates an unambiguous mapping of the surfaces is used to define the light map or coefficient textures. Using the tangent space of the second texture coordinate set, calculated from the UV directions of this set, to represent the irradiance would result in the fact that the tangent space normal maps reside in a different tangent space than the coefficient maps.

Fortunately, both texture coordinate sets share the same vertices and normals as well as interpolation in the rendering pipeline. By simply using the tangent and bitangent of the normal map tangent space in the irradiance texture coordinates during the precomputation of the irradiance, a tangent space using the irradiance texture coordinate set is defined that is exactly aligned in object space with the normal map tangent space. This step is allowed because we only need the local directions of the tangent space during the run-time evaluation and not its position. The looked up normal can be used directly to evaluate the weighted basis functions without any additional data or transformations.

Effectively, the irradiance texture coordinate set is only used to define the surface points where the irradiance is calculated and cached, while the normal map tangent space defines the tangent and bitangent for both spaces, resulting in a correctly aligned irradiance signal relative to the looked up normal. 


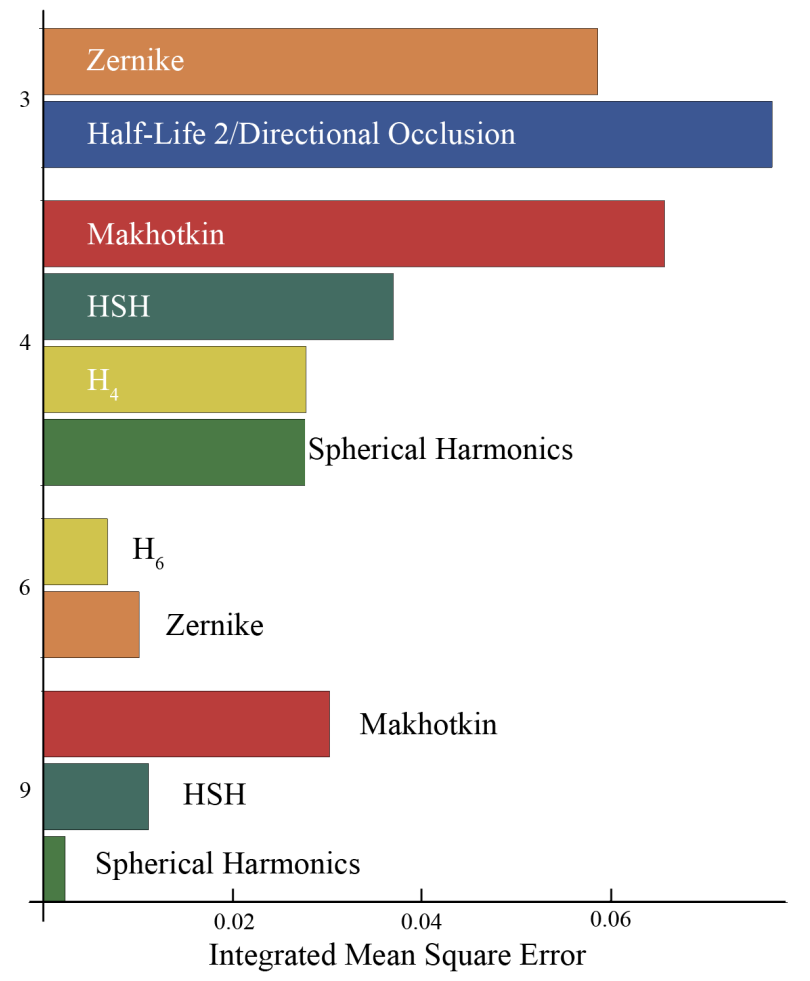

Figure 3: Average of the integrated mean square error over 10,000 random irradiance functions grouped by number of coefficients.

\section{Error Analysis and Results}

Since any hemispherical basis such as described in Section 2 can carry an approximation of a hemispherical irradiance signal, we can directly compare the $\mathcal{H}$-basis to any other hemispherical basis by substituting it for the $\mathcal{H}$-basis in the precomputation as well as in the run-time evaluation to determine their accuracy. As an error metric, we use the integrated mean square error

$$
\mathrm{IMSE}=\int_{\Omega_{+}}\left(\sum_{i=1}^{n} c_{i} B_{i}(\vec{n})-E(\vec{n})\right)^{2} d \vec{n}
$$

where $E(\vec{n})$ is the fully correct irradiance signal and $c_{i} B_{i}$ the corresponding weighted basis function of each basis. The IMSE of all bases with different numbers of coefficients averaged over 10,000 random irradiance signals is shown in Figure 3.

As can bee seen, the $\mathcal{H}$-basis, both with 4 basis functions can deliver the same error as least square projected linear Spherical Harmonics while being an orthogonal basis. With 6 coefficients the lowest error for the number of coefficients used, leveraging the same advantages as Spherical Harmonics. Interestingly, the Zernike-basis is also showing a low error and has a lower error with 3 coefficients than the Half-Life 2 basis, though 3 coefficients are not enough for an acceptable representation as can be seen in Figure 4.

Although the average error of the Zernike basis is only slightly higher than $H^{6}$, it has a much higher evaluation cost due to its non-polynomial basis functions. An evaluation of this basis with 6 basis functions contains two square roots and 4 divisions. Also, the Zernike basis requires 6 functions while the $\mathcal{H}$-basis already allows

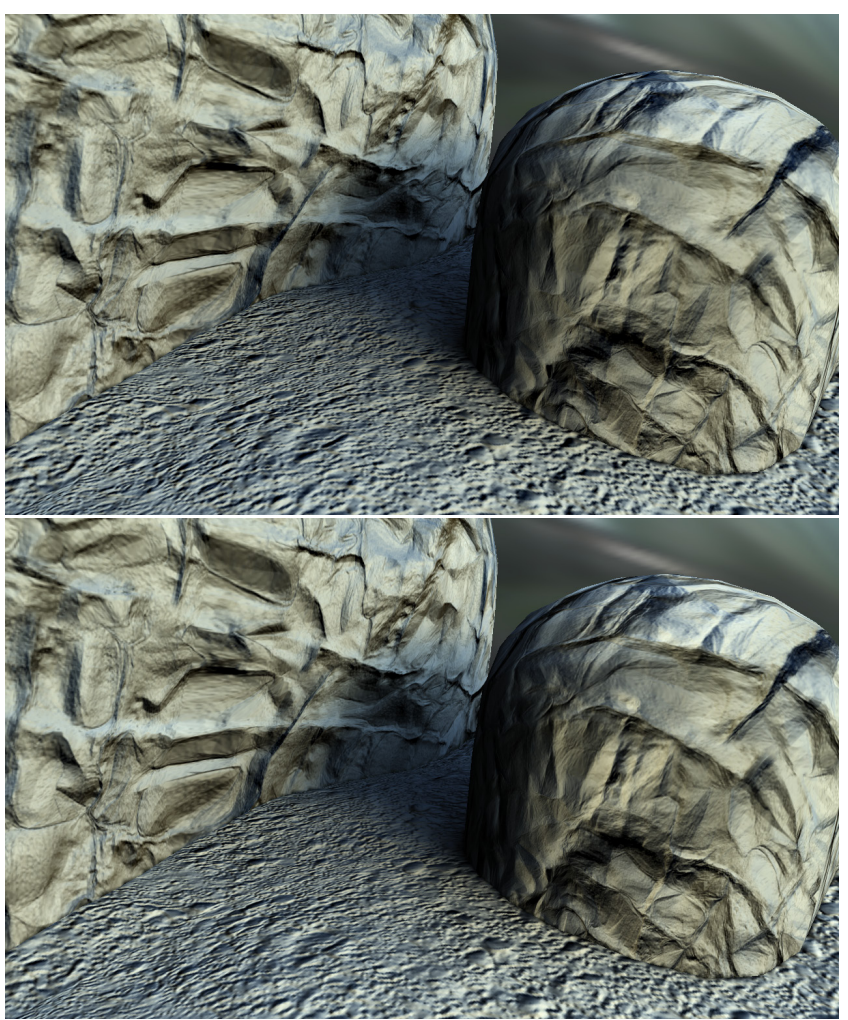

Figure 5: Detail of a game scene without albedo texturing using $\mathrm{H}_{4}$ (upper) and the more accurate $\mathrm{H}_{6}$ (lower) basis. The differences are marginal, while the more accurate $H_{6}$ basis shows slightly more defined detail.

a good representation with 4 basis functions. The similar effectiveness in representation of the Zernike basis and the $\mathcal{H}$-basis can be explained by the fact that the basis functions have a similar shape, besides sharing two basis functions, namely the constant function $H^{1}$ and $H^{3}$, which is also true for HSH. Comparing the $\mathcal{H}$-basis to bases with 9 coefficients, only quadratic Spherical Harmonics have a lower error.

Figure 4 shows a direct comparison of all bases using two or three bands under the same lighting conditions. The used lighting is an HDR environment map with the sun at grazing angle, which poses a worst case situation because the irradiance changes strongly in the longitudinal as well as latitudinal parameter. For practical purposes, though the error is still higher than quadratic Spherical Harmonics, $H_{6}$ delivers an accurate representation as can be seen in the absolute difference images of both Spherical Harmonics and $H_{6}$.

A typical game scene using $H_{6}$ can bee seen in Figure 1, which also shows the difference between traditional light mapping and irradiance normal mapping. Figure 5 shows a close-up depicting the difference between $H_{4}$ and $H_{6}$. As can be seen, the perceptual difference between $H_{4}$ and $H_{6}$ is marginal and, due to the orthogonality of the basis, distributed over the hemisphere, which makes $\mathrm{H}_{4}$ the most efficient choice. Using only two more coefficients with the $H_{6}$ basis, the result is slightly more pronounced since the longitudinal accuracy is increased, and can deliver a numerical accuracy comparable to quadratic Spherical Harmonics in case the basis is used for other purposes than irradiance normal mapping or if a higher accuracy is desired. 


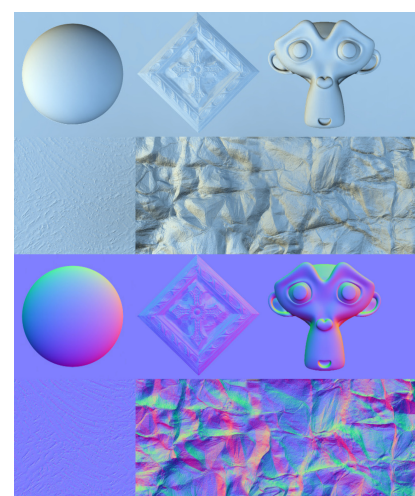

Ground Truth

Normal map

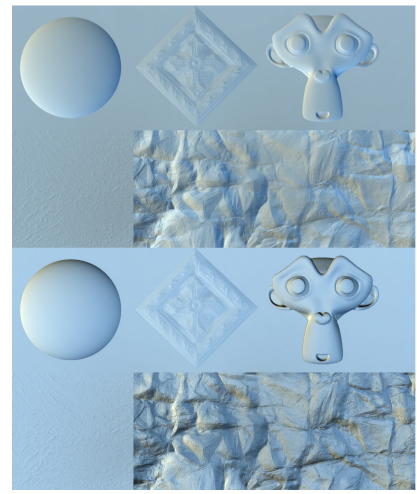

Zernike, 3 coefficients 6 coefficients

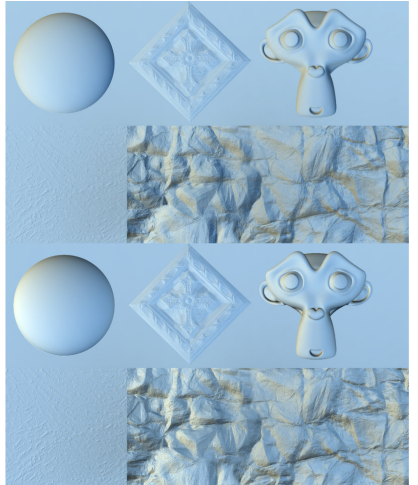

Spherical Harmonics, 2 bands 3 bands

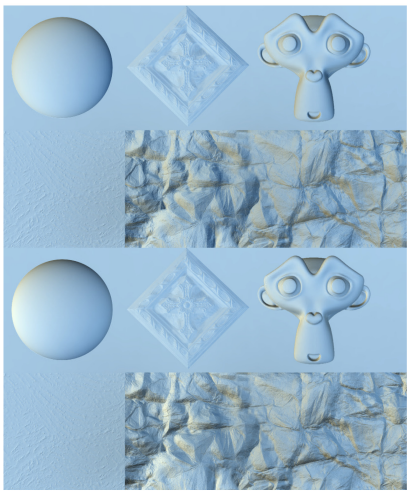

$\mathrm{H}_{4}$

$\mathrm{H}_{6}$

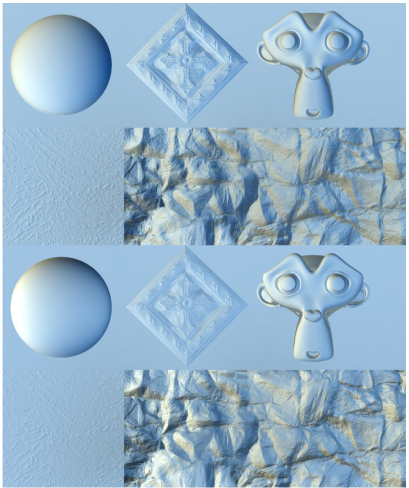

HSH, 2 bands

3 bands

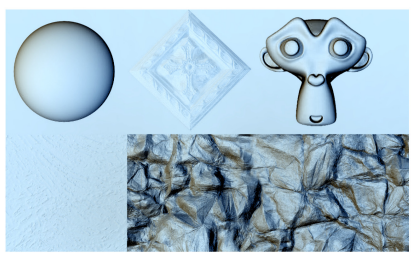

Half-Life 2/Dir. Occlusion

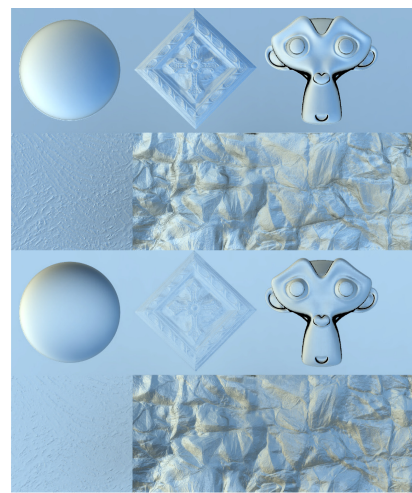

Makhotkin, 2 bands

3 bands
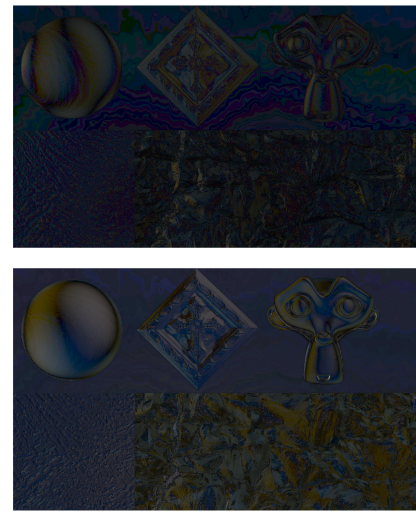

Diff. Ground Truth - SH 3 bands Diff. Ground Truth - $\mathrm{H}_{6}$

Figure 4: Comparison of all hemispherical bases with different number of bands representing the same irradiance signal. The lower right images show the absolute difference of quadratic Spherical Harmonics and $H_{6}$ to the ground truth.

\section{Conclusion and Future Work}

We have designed a new hemispherical basis, called $\mathcal{H}$-basis, which is optimized for representing a directional irradiance signal on the hemisphere for the purpose of irradiance normal mapping. It is the best option for a low number of coefficients without sacrificing the advantages of orthogonal polynomial basis systems, being the fastest basis to evaluate and having the lowest error to coefficients ratio. Analogous to other bases, the number of coefficients increases the accuracy, keeping the possibility to change the number of coefficients at run-time to create shader levels-of-detail or to fit the number of coefficients to the given resources.

We showed that 4 coefficients are sufficient for a perceptually acceptable irradiance representation and 6 coefficients for a numerically accurate representation. Besides directional irradiance signals, the $\mathcal{H}$-basis is also a good choice to represent other lowfrequency signals on the hemisphere and any signal can be directly projected into the $\mathcal{H}$-basis due to its orthogonality. Specifically, the $\mathcal{H}$-basis can replace the Half-Life 2 basis in methods such as [Sloan 2006] and [Habel et al. 2007], delivering a far higher accuracy with just one coefficient more.

The fact that no explicit orthogonalization has been used to derive the basis functions hints at an undiscovered polynomial orthonormal basis on the hemisphere that is the direct equivalent of Spheri- cal Harmonics, sharing most of its attributes.

\section{References}

BASRI, R., AND JACOBS, D. 2000. Lambertian reflectance and linear subspaces. Tech. rep., Jerusalem, Israel, Israel.

Chen, H., AND LIU, X. 2008. Lighting and material of halo 3. In SIGGRAPH '08: ACM SIGGRAPH 2008 classes, ACM, New York, NY, USA, 1-22.

Gautron, P., Krivánek, J., Pattanaik, S. N., and BouaTOUCH, K. 2004. A novel hemispherical basis for accurate and efficient rendering. In Rendering Techniques, 321-330.

GreEn, R. 2003. Spherical harmonic lighting: The gritty details. Archives of the Game Developers Conference (March).

GREEN, C. 2007. Efficient self-shadowed radiosity normal mapping. In SIGGRAPH '07: ACM SIGGRAPH 2007 courses, ACM, New York, NY, USA, 1-8.

Groemer, H. 1996. Geometric Applications of Fourier Series and Spherical Harmonics. Cambridge University Press.

Habel, R., Kusternig, A., And Wimmer, M. 2007. Physically based real-time translucency for leaves. In Rendering Tech- 
niques 2007 (Proceedings Eurographics Symposium on Rendering), Eurographics Association, J. Kautz and S. Pattanaik, Eds., Eurographics, 253-263.

JENSEN, H. W. 1996. Global illumination using photon maps. In Proceedings of the eurographics workshop on Rendering techniques '96, Springer-Verlag, London, UK, 21-30.

KAJIYA, J. T. 1986. The rendering equation. In SIGGRAPH '86: Proceedings of the 13th annual conference on Computer graphics and interactive techniques, ACM, New York, NY, USA, 143150 .

Koenderink, J. J., Doorn, A. J. V., And StaVRidi, M. 1996. Bidirectional reflection distribution function expressed in terms of surface scattering modes. In ECCV '96: Proceedings of the 4th European Conference on Computer Vision-Volume II, Springer-Verlag, London, UK, 28-39.

LAM, P.-M., LeUng, C.-S., AND Wong, T.-T. 2006. Noiseresistant fitting for spherical harmonics. IEEE Transactions on Visualization and Computer Graphics 12, 2, 254-265.

Makhotkin, O. A. 1996. Analysis of radiative transfer between surfaces by hemispherical harmonics. Journal of Quantitative Spectroscopy and Radiative Transfer 56, 6, 869-879.

McTaggert, G. 2004. Half-Life 2/Valve Source Shading. Tech. rep., Valve Corporation.

RAMAMOORTHI, R., AND HANRAHAN, P. 2001. An efficient representation for irradiance environment maps. In SIGGRAPH '01: Proceedings of the 28th annual conference on Computer graphics and interactive techniques, ACM, New York, NY, USA, 497500 .

Sansone, G. 1991. Orthogonal Functions. Dover Publications.

SCHRÖDER, P., AND SWELDENS, W. 1995. Spherical wavelets: efficiently representing functions on the sphere. In SIGGRAPH '95: Proceedings of the 22nd annual conference on Computer graphics and interactive techniques, ACM, New York, NY, USA, 161-172.

Sloan, P.-P., KaUtz, J., And SNyder, J. 2002. Precomputed radiance transfer for real-time rendering in dynamic, lowfrequency lighting environments. In SIGGRAPH '02: Proceedings of the 29th annual conference on Computer graphics and interactive techniques, ACM, New York, NY, USA, 527-536.

Sloan, P.-P., Hall, J., Hart, J., AND SnYder, J. 2003. Clustered principal components for precomputed radiance transfer. ACM Trans. Graph. 22, 3, 382-391.

SloAN, P.-P. 2006. Normal mapping for precomputed radiance transfer. In I3D '06: Proceedings of the 2006 symposium on Interactive $3 D$ graphics and games, ACM, New York, NY, USA, 23-26.

SlOAN, P.-P. 2008. Stupid spherical harmonics (sh) tricks.

\section{Appendix A}

Transformation matrix $T_{h}^{4} \mathrm{x}$ for $l=4$ coefficients arranged in a vector of the form $s_{-4}^{4} . . s_{4}^{4}$.

$$
\left(\begin{array}{ccccccccc}
0 & 0 & 0 & 0 & 0 & 0 & 0 & 0 & 0 \\
0 & 0 & 0 & -\frac{\sqrt{15}}{16} & 0 & 0 & 0 & 0 & 0 \\
0 & 0 & 0 & 0 & -\frac{1}{8} \sqrt{\frac{3}{2}} & 0 & 0 & 0 & 0 \\
0 & 0 & 0 & 0 & 0 & -\frac{\sqrt{15}}{16} & 0 & 0 & 0 \\
0 & 0 & 0 & 0 & 0 & 0 & 0 & 0 & 0 \\
0 & 0 & 0 & 0 & 0 & 0 & 0 & 0 & 0
\end{array}\right)
$$

